\title{
Tables of Values of the Modified Mathieu Functions
}

\author{
By E. T. Kirkpatrick
}

1. Introduction. Mathieu functions are encountered in physical problems involving elliptical boundaries. When the method of separation of variables is applied to the wave equation or the diffusion equation expressed in elliptical coordinates, there result $[1$, p. 170] the Mathieu and modified Mathieu equations,

$$
\begin{aligned}
& \frac{d^{2} y}{d u^{2}}+(a-2 q \cos 2 u) y=0 \\
& \frac{d^{2} y}{d u^{2}}-(a-2 q \cosh 2 u) y=0 .
\end{aligned}
$$

The notation used is that of Goldstein, Ince and McLachlan. Solutions to the modified Mathieu equation (2) can be assumed to be of the form

$$
\begin{aligned}
& y=C_{2 n}(u, q)=\sum_{r=0}^{\infty} A_{2 r}^{(2 n)} \cosh 2 r u \\
& y=C_{2 n+1}(u, q)=\sum_{r=0}^{\infty} A_{2 r+1}^{(2 n+1)} \cosh (2 r+1) u \\
& y=S_{\left(i^{2}+1\right.}(u, q)=\sum_{r=0}^{\infty} B_{2 r+1}^{(2 n+1)} \sinh (2 r+1) u \\
& y=S_{\left(y_{n+2}\right.}(u, q)=\sum_{r=0}^{\infty} B_{2 r+2}^{(2 n+2)} \sinh (2 r+2) u .
\end{aligned}
$$

When the above infinite hyperbolic series are substituted into equation (2), recurrence relationships may be derived for allowable values of the characteristic numbers $a_{2 n+p}(p=0$ or 1$)$ for the even functions, or $b_{2 n+s}(s=1$ or 2$)$ for the odd functions for a given value of $q$. Recurrence equations also give the allowable values of the Fourier coefficients $A_{2 r+p}^{(2 n+p)}$ and $B_{2 r+s}^{(2 n+\infty)}$

The formulas for both the characteristic numbers and the Fourier coefficients associated with each type of solution $[1$, p. 29, 37] are given below:

For $y=C e_{2 n}(u, q)$

$$
\begin{array}{rlrl}
a_{2 n} & =\frac{2 q^{2}}{a-2^{2}-\frac{q^{2}}{a-4^{2}-} \frac{q^{2}}{a-6^{2}-} \cdots \frac{q^{2}}{a-(2 r)^{2}-} \cdots} & \\
v_{2 r-2}=\frac{q}{a-(2 r)^{2}-q v_{2 r}}, & r \geqq 2 \\
v_{0}=\frac{2 q}{a-(2 r)^{2}-q v_{2}}, & r=1
\end{array}
$$

Received August 18, 1958; revised September 28, 1959. 
where

$$
v_{2 n-2}=\frac{A_{2 r}^{(2 n i}}{A_{2 r-2}^{(2 n)}} .
$$

For $y=C e_{2 n+1}(u, q)$

$$
a_{2 n+1}=1+q+\frac{q^{2}}{a-3^{2}-} \frac{q^{2}}{a-5^{2}-\ldots} \frac{q^{2}}{a-(2 r+1)^{2}-\ldots}
$$

$$
v_{2 r-1}=\frac{q}{a-(2 r+1)^{2}-q v_{2 r+1}} \text {, }
$$

$$
v_{1}=\frac{a-1-q}{q}
$$$$
r=1 \text {. }
$$

$$
\text { For } y_{2 n+1}=S e_{2 n+1}(u, q)
$$

(6a). $\quad b_{2 n+1}=1-q+\frac{q^{2}}{a-3^{2}-} \frac{q^{2}}{a-5^{2}-} \cdots \frac{q^{2}}{a-(2 r+1)^{2}-\cdots}$

$$
\begin{array}{rlrl}
v_{2 r+1} & =\frac{q}{a-(2 r+1)^{2}-q v_{2 r+1}}, & r \geqq 2 \\
v_{1}=\frac{a-1+q}{q}, & r=1 .
\end{array}
$$

For $y_{2 n+2}=\operatorname{Se}_{2 n+2}(u, q)$

$$
\begin{aligned}
& b_{2 n+2}=4+\frac{q^{2}}{a-4^{2}-} \frac{q^{2}}{a-6^{2}-} \cdots \frac{q^{2}}{a-(2 r)^{2}-} \ldots \\
& v_{2 r-2}=\frac{q}{a-(2 r)^{2}-q v_{2 r}}, \\
& r \geqq 3 \\
& v_{2}=\frac{a-4}{q}, \\
& r=\ddot{2} \text {. }
\end{aligned}
$$

As a consequence $[1$, p. 24] of the Goldstein-Ince normalization

(8) $\int_{0}^{2 \pi} y^{2} d u=\pi$, then for $y=C e_{2 n}(u, q), \quad 1=2\left[A_{0}^{(2 n)}\right]^{2}+\sum_{r=1}^{\infty}\left[A_{2 r}^{(2 n)}\right]^{2}$

and for the remaining three types,

$$
1=\sum_{r=0}^{\infty}\left[A_{2 r+1}^{(2 n+1)}\right]^{2}=\sum_{r=0}^{\infty}\left[B_{2 r+1}^{(2 n+1)}\right]^{2}=\sum_{r=0}^{\infty}\left[B_{2 r+2}^{(2 n+2)}\right]^{2} .
$$

By dividing equations (8) and (9) through by $\left[A_{0}{ }^{(2 n)}\right]^{2}$ there are obtained equations of the form

$$
\begin{gathered}
{\left[\frac{1}{A_{0}^{(2 n)}}\right]^{2}=2+\left[\frac{A_{2}^{(2 n)}}{A_{0}^{(2 n)}}\right]^{2}+\left[\frac{A_{4}^{(2 n)}}{A_{0}^{(2 n)}}\right]^{2}+\left[\frac{A_{0}^{(2 n)}}{A_{0}^{(2 n)}}\right]^{2}+\cdots} \\
=2+v_{0}^{2}+\left(v_{2} \cdot v_{0}\right)^{2}+\left(v_{4} \cdot v_{2} \cdot v_{0}\right)^{2}+\cdots
\end{gathered}
$$

2. Computation of the Modified Mathieu Functions. For a given value of $q$, the characteristic number is found by using a trial, error and interpolation method 
using the appropriate transcendental continued fraction of the form of equation (ta). The computation was generally started with the 21 st term equal to zero, and then the 20th, 19th, etc., terms were computed in turn.

The $v_{2 r}$ are all known from equations of the type (4b) and (4c) above and therefore $A_{0}$ can be evaluated from equation (10). Equation (4d) will then give the value of the remaining $A_{2 r}$. The value of the modified Mathieu function can then be computed by summing the appropriate hyperbolic series.

In [2], E. L. Ince has given tables for the characteristic numbers, Fourier coefficients and values of the Mathicu functions satisfying equation (1). The method used by Ince was to apply the above formulas; the same method was used by the author to evaluate the modified Mathieu functions satisfying equation (2). The numerical work was accomplished by using an IBM 650 Digital Computer and the Wolontis. Interpretive System of coding. The characteristic numbers and the Fourier coefficients are the same for both the ordinary and the modified functions. However, the problem of summing the hyperbolic series is much more difficult than that of summing the trigonometric series. Whereas the cos $2 r u$ term is bounded by plus or minus one, the $\sinh 2 r u$ and cosh $2 r u$ approach infinity as $r$ tends to infinity. Therefore many more terms of the hyperbolic series must be considered before it, is possible to generate a product $A_{2 r}^{(2 n)} \cosh 2 r u$ which will be negligibly small.

As more terms are taken, difficulties in computation are encountered. The $v_{2 r}$ can be made as small as desired by choosing $r$ sufficiently large. In most cases after the characteristic number had been found by using the appropriate transcendental continued fraction, the computation of the Fourier coefficients was started by letting $v_{40}=0$, and then compute the $v_{38}, v_{36}, \cdots v_{0}$, in turn. As these values were computed, they were stored and used to form the products $v_{0}^{2},\left(v_{2} \cdot v_{0}\right)^{2}$, etc., for equation (10). It is to be noted that the smallest number which can be computed using the Wolontis Interpretive System is $10^{-50}$. At first consideration this may not appear to be a serious limitation. However, in order to obtain, for example, fifteen coefficients up to $A_{28}^{(2 n)}$, from the relations $A_{2 r+2}=v_{2 r} A_{2 r}$, the $v_{28}$ must be available. For it to be available the product of $\left(v_{28} \cdot v_{24} \cdots \cdots v_{0}\right)^{2}$ must be greater than $10^{-50}$, or the unsquared value must be greater than $10^{-25}$. Except for $v_{0}, v_{2}$ and $v_{4}$ which may sometimes be greater than one, all the remaining values are less than one. In fact, for convergence, $v_{2 r} \rightarrow 0$ as $r \rightarrow \infty$. If, for example, the "average" size of the $v_{2 r}$ is of the order of $10^{-2}$, only twelve $v_{2 r}$ can be multiplied together before their product becomes less than $10^{-25}$. Therefore, for this example, the maximum number of coefficients that can be generated will be twelve or thirteen. A machine error stop was avoided by counting the products as they were formed and at the same time checking their magnitude. The maximum number of coefficients generated was twenty; that is, up to and including coefficient $A_{38}^{(2 n)}$. The number of coefficients generated will depend on the values of $q$ and order $2 n$ being considered.

3. Description of Computer Programs. A computer program was developed for the IBM 650 Digital Computer which will compute the characteristic numbers and Fourier coefficients and will sum the appropriate series in order to find the value of either the ordinary or the modified Mathieu function. A complete description of this program titled "A Program for the Computation of Mathieu and Modified Mathieu Functions" is available from the author and will be published separately. 
In order to ensure tive figure accuracy for parameters in the ranges $0 \leqq q \leqq$ $20,0 \leqq n \leqq 3$ and $0 \leqq u \leqq 1.0$, the program can use a maximum of twenty terms. For parameters outside the given ranges, the accuracy is unknown. However, the user can estimate the accuracy by noting the magnitude of the last term added to the series.

4. Discussion of Tables. Tables 1-12 are provided herein for values of the four types of modified Mathieu function $C e_{2 n}(u, q), C e_{2 n+1}(u, q), S e_{2 n+1}(u, q)$ and $S e_{2 n+2}(u, q)$, for the ranges $q=1(1) 10(2) 20, u=0.1(0.1) 1.0$ and $n=0,1,2$. The Fourier coefficients computed in order to obtain these values were compared with existing tables [1] for over 150 values, and no discrepancy was found in the first seven digits of any coefficient generated. Also, the magnitude of the last term added in the series was noted to ensure the accuracy of the last position published.

5. Acknowledgments. The author wishes to express his appreciation to $\mathrm{Dr}$. W. Stokey of the Mechanical Engineering Department of Carnegie Institute of Technology and to the staff of the Carnegie Institute of Technology Computation Center for their helpful advice and criticism. The computations contained herein were made possible through the use of the facilities of the Computation Center in accordance with the policy granting free computer time to all unsponsored faculty and graduate research.

Mechanical Engineering Department

University of Toledo

Toledo, Ohio

1. H. W. Mclachlan, Theory and Application of Mathieu Functions, Oxford University Press, London, 1947.

2. E. L. Ince, "Tables of the elliptical cylinder functions," Roy. Soc., Edinburgh, Proc., v. 52,1932 , p. $355-423$.

TABLE 1

Values of Modified Mathieu Function Ce $e_{0}(u, q)$

$\begin{array}{cccccc}q & u=0.1 & u=0.2 & u=0.3 & u=0.4 & u=0.5 \\ 1 & .38010 & .36588 & .34209 & .3086 .3 & .26554 \\ 2 & .19704 & .18047 & .15340 & .11679 & .07224 \\ 3 & .10989 & .09505 & .07142 & .04076 & .00576 \\ 4 & .06564 & .05329 & .03412 & .01034 & -.01491 \\ 5 & .04130 & .03127 & .01612 & -.00179 & -.01932 \\ 6 & .02702 & .01895 & .00709 & -.00623 & -.01807 \\ 7 & .01822 & .01174 & .00249 & -.00734 & -.01515 \\ 8 & .01259 & .00738 & .00017 & -.00705 & -.01206 \\ 9 & .00887 & .00468 & -.00094 & -.00624 & -.00931 \\ 10 & .00636 & .00298 & -.00142 & -.00529 & -.00705 \\ 12 & .00341 & .00118 & -.00153 & -.00355 & -.00388 \\ 14 & .00190 & .00042 & -.00126 & -.00229 & -.00203 \\ 16 & .00110 & .00010 & -.00095 & -.00144 & -.00101 \\ 18 & .00065 & -.00002 & -.00069 & -.00089 & -.00045 \\ 20 & .00040 & -.00007 & -.00049 & -.00054 & -.00016 \\ q & u=0.6 & u=0.7 & u=0.8 & u=0.9 & u=1.0 \\ 1 & .21308 & .15200 & .08378 & .01097 & -.06241 \\ 2 & .02222 & -.02961 & -.07835 & -.11775 & -.14074 \\ 3 & -.02984 & -.06129 & -.08309 & -.08980 & -.07753\end{array}$


TABLe 1 (Continued)

$\begin{array}{rrrrrr}4 & -.03764 & -.05332 & -.05766 & -.04792 & -.02464 \\ 5 & -.03275 & -.03843 & -.03382 & -.01887 & .00267 \\ 6 & -.02529 & -.02528 & -.01712 & -.00270 & .01269 \\ 7 & -.01841 & -.01551 & -.00677 & .00478 & .01379 \\ 8 & -.01290 & -.00880 & -.00094 & .00728 & .01122 \\ 9 & -.00876 & -.00443 & .00199 & .00722 & .00773 \\ 10 & -.00576 & -.00172 & .00319 & .00606 & .00457 \\ 12 & -.00219 & .00073 & .00314 & .00319 & .00054 \\ 14 & -.00055 & .00127 & .00211 & .00113 & -.00092 \\ 16 & .00011 & .00112 & .00116 & .00007 & -.00106 \\ 18 & .000332 & .00081 & .00051 & -.00033 & -.00074 \\ 20 & .00034 & .00052 & .00014 & -.00039 & -.00038\end{array}$

TABLE 2

Values of Modified Mathieu Function $\mathrm{Ce}_{1}(u, q)$

\begin{tabular}{|c|c|c|c|c|c|}
\hline$q$ & $u=0.1$ & $u=0.2$ & $u=0.3$ & $u=0.4$ & $u=0.5$ \\
\hline 1 & .855996 & .85 .372 & .84884 & .83954 & .82334 \\
\hline 2 & .67799 & . $.6(6081$ & . 63071 & .58567 & .52324 \\
\hline 3 & .50011 & . 47318 & .42746 & .36215 & .27703 \\
\hline 4 & .35\%310 & .32216 & .27089 & .20034 & .11323 \\
\hline 5 & $.246 j 12$ & .21525 & .16 .523 & .09889 & .02144 \\
\hline t & . 17252 & . $14: 384$ & .09841 & .04040 & -.02332 \\
\hline 7 & .12249 & .09685 & .05717 & .00848 & -.04153 \\
\hline 8 & .08824 & .06581 & .03192 & -.00795 & -.04594 \\
\hline 9 & $.06445)$ & .04509 & .01652 & -.01561 & -.04369 \\
\hline 10 & .047699 & .03109 & .00721 & -.01840 & -.03863 \\
\hline 12 & $.026 ; 97$ & $.0149) 3$ & -.00151 & -.01739 & -.02697 \\
\hline 14 & .01582 & .0071:3 & -.00408 & -.01366 & -.01732 \\
\hline 16 & (00!5:5) & $.00: 329$ & -.004333 & -.00994 & -.01054 \\
\hline 18 & .00591 & .00138 & -.00379 & -.00696 & -.00612 \\
\hline 20 & $.00: 37: 3$ & .00044 & -.00306 & -.00475 & -.00337 \\
\hline$q$ & $u=0.6$ & $u=0.7$ & $u=0.8$ & $u=0.9$ & $u=1.0$ \\
\hline 1 & .796907 & $.75(6.52)$ & .69758 & .61571 & .50717 \\
\hline 2 & $.+410: 3$ & .337:53 & .21334 & .07268 & -.07467 \\
\hline 3 & $.17: 3 \cdot 4 !)$ & $.05 \overline{5} 84$ & -.06712 & -.18088 & -.26516 \\
\hline 4 & .01512 & $-.08+42$ & -.17102 & -.22623 & -.23096 \\
\hline 5 & - .0:58:58 & -.12880 & -.17365 & -.17745 & -.13069 \\
\hline 6 & -.08277 & -.12515 & -.13706 & -.10917 & -.04351 \\
\hline 7 & $-.0826 i i 3$ & $-.10: 3: 28$ & -.09387 & -.05217 & .01053 \\
\hline 8 & -.07238 & -.07784 & -.05696 & -.01349 & .03573 \\
\hline 9 & $-.0591+t$ & -.05494 & -.02954 & .00899 & .0419 \\
\hline 10 & $-.04 t i 21$ & -.03648 & -.01097 & .01974 & .037 \\
\hline 12 & $-.0256+1$ & -.01254 & .00704 & .02185 & .0202 \\
\hline 14 & -.01264 & -.00112 & .01088 & $.01 \pm 63$ & .00571 \\
\hline 16 & -.00526 & $.00: 325$ & .00923 & .00728 & -.0017 \\
\hline 18 & $-.001+2$ & $.00+21$ & .00625 & .00231 & -.004 \\
\hline 20 & $.000: 37$ & .00374 & .00359 & -.00032 & \\
\hline
\end{tabular}

TABLE 3

Values of Modified Mathieu Function $\mathrm{Ce}_{2}(u, q)$

$\begin{array}{lccccc}q & u=0.1 & u=0.2 & u=0.3 & u=0.4 & u=0.5 \\ 1 & 1.0988 & 1.1373 & 1.2008 & 1.2883 & 1.3974 \\ 2 & 1.0549 & 1.0724 & 1.0988 & 1.1297 & 1.1585\end{array}$


TABLE 3 (Continued)

$\begin{array}{rrrrrr}3 & 0.96379 & 0.96299 & 0.95773 & 0.94205 & 0.90754 \\ 4 & 0.85071 & 0.83405 & 0.80203 & 0.74858 & 0.66614 \\ 5 & 0.72581 & 0.69617 & 0.64300 & 0.56150 & 0.44693 \\ 6 & 0.59925 & 0.56021 & 0.49256 & 0.39375 & 0.26316 \\ 7 & 0.48147 & 0.43689 & 0.36159 & 0.25581 & 0.12340 \\ 8 & 0.37974 & 0.33303 & 0.25593 & 0.15146 & 0.02752 \\ 9 & 0.29657 & 0.25032 & 0.17560 & 0.07791 & -0.03165 \\ 10 & 0.23086 & 0.18674 & 0.11699 & 0.02902 & -0.06385 \\ 12 & 0.14063 & 0.10314 & 0.04636 & -0.01995 & -0.08086 \\ 14 & 0.08716 & 0.05686 & 0.01298 & -0.03411 & -0.07028 \\ 16 & 0.05511 & 0.03123 & -0.00175 & -0.033933 & -0.05319 \\ 18 & 0.03549 & 0.01694 & -0.00746 & -0.02879 & -0.03736 \\ 20 & 0.02324 & 0.00892 & -0.00894 & -0.02268 & -0.02491 \\ q & u=0.6 & u=0.7 & 4=0.8 & u=0.9 & u=1.0 \\ 1 & 1.5243 & 1.6624 & 1.8011 & 1.9253 & 2.0134 \\ 2 & 1.1753 & 1.1676 & 1.1197 & 1.0143 & 0.83594 \\ 3 & 0.84378 & 0.73947 & 0.58490 & 0.37624 & 0.12180 \\ 4 & 0.54718 & 0.38688 & 0.18749 & -0.03624 & -0.25175 \\ 5 & 0.29722 & 0.11677 & -0.07877 & -0.25853 & -0.37664 \\ 6 & 0.10531 & -0.06602 & -0.22436 & -0.33044 & -0.34055 \\ 7 & -0.02480 & -0.16773 & -0.27310 & -0.30268 & -0.22806 \\ 8 & -0.10013 & -0.20621 & -0.25818 & -0.22648 & -0.10465 \\ 9 & -0.13427 & -0.20357 & -0.21119 & -0.14039 & -0.00653 \\ 10 & -0.14150 & -0.17901 & -0.15482 & -0.06588 & 0.05530 \\ 12 & -0.11743 & -0.11198 & -0.05854 & 0.02440 & 0.08984 \\ 14 & -0.08057 & -0.05594 & -0.00279 & 0.05087 & 0.06479 \\ 16 & -0.04921 & -0.02021 & 0.02043 & 0.04520 & 0.02999 \\ 18 & -0.02702 & -0.00098 & 0.02511 & 0.02973 & 0.00504 \\ 20 & -0.01284 & 0.00753 & 0.02147 & 0.01512 & -0.00745\end{array}$

TABLE 4

Values of Modified Mathieu Function $\mathrm{Ce}_{3}(u, q)$

$\begin{array}{rccccc}q & u=0.1 & u=0.2 & u=0.3 & u=0.4 & u=0.5 \\ 1 & 1.1052 & 1.2213 & 1.4222 & 1.7189 & 2.1258 \\ 2 & 1.1586 & 1.2504 & 1.4052 & 1.6250 & 1.9097 \\ 3 & 1.1812 & 1.2486 & 1.3585 & 1.5061 & 1.6816 \\ 4 & 1.1654 & 1.2097 & 1.2780 & 1.3609 & 1.4428 \\ 5 & 1.1197 & 1.1428 & 1.1740 & 1.2010 & 1.2055 \\ 6 & 1.0538 & 1.0580 & 1.0563 & 1.0356 & 0.97750 \\ 7 & 0.97392 & 0.96113 & 0.93084 & 0.87011 & 0.76270 \\ 8 & 0.88402 & 0.85635 & 0.80166 & 0.70851 & 0.56459 \\ 9 & 0.78762 & 0.74742 & 0.67297 & 0.55533 & 0.38747 \\ 10 & 0.68880 & 0.63876 & 0.54956 & 0.41562 & 0.23594 \\ 12 & 0.50147 & 0.44023 & 0.33678 & 0.19314 & 0.02033 \\ 14 & 0.34898 & 0.28634 & 0.18502 & 0.05393 & -0.08713 \\ 16 & 0.23815 & 0.18014 & 0.09012 & -0.01830 & -0.12097 \\ 18 & 0.16205 & 0.11131 & 0.03578 & -0.04848 & -0.11669 \\ 20 & 0.11083 & 0.06792 & 0.00673 & -0.05603 & -0.09738 \\ q & u=0.6 & u=0.7 & u=0.8 & u=0.0 & u=1.0 \\ 1 & 2.6602 & 3.3395 & 4.1775 & 5.1777 & 6.3233 \\ 2 & 2.2548 & 2.6466 & 3.0569 & 3.4362 & 3.7080 \\ 3 & 1.8671 & 2.0337 & 2.138 .3 & 2.1234 & 1.9238 \\ 4 & 1.4998 & 1.4989 & 1.3999 & 1.1630 & 0.76444\end{array}$


TABle 4 (Continued)

$\begin{array}{rrrrrr}5 & 1.1629 & 1.0441 & 0.82247 & 0.48677 & 0.06084 \\ 6 & 0.86034 & 0.66443 & 0.38210 & 0.03295 & -0.32004 \\ 7 & 0.59306 & 0.35396 & 0.05843 & -0.24680 & -0.47537 \\ 8 & 0.36249 & 0.10894 & -0.16367 & -0.38955 & -0.47787 \\ 9 & 0.17129 & -0.07282 & -0.29711 & -0.42722 & -0.38634 \\ 10 & 0.02180 & -0.19459 & -0.35593 & -0.39057 & -0.25026 \\ 12 & -0.15498 & -0.28704 & -0.31889 & -0.21098 & 0.01050 \\ 14 & -0.2039 .5 & -0.2516 .5 & -0.19309 & -0.03416 & 0.14269 \\ 16 & -0.18368 . & -0.17205 & -0.07499 & 0.06593 & 0.15185 \\ 18 & -0.13945 & -0.096336 & 0.00146 & 0.09533 & 0.10267 \\ 20 & -0.0948 .3 & -0.04108 & 0.03835 & 0.08428 & 0.04596\end{array}$

TABLE 5

Values of Modified Mathieu Function $\mathrm{Ce}_{4}(u, q)$

\begin{tabular}{|c|c|c|c|c|c|}
\hline$q$ & $u=0.1$ & $u=0.2$ & $u=0.3$ & $u=0.4$ & $\begin{array}{l}u=0.5 \\
2\end{array}$ \\
\hline 1 & 1.1086 & $\begin{array}{l}1.3389 \\
1.3447\end{array}$ & 1.7569 & 2.4173 & 3.4036 \\
\hline 2 & 1.1402 & 1.3447 & 1.7094 & 2.2701 & 3.0767 \\
\hline 3 & 1.1750 & 1.3539 & 1.6668 & 2.1337 & 2.7777 \\
\hline 4 & 1.2090 & 1.3622 & 1.6246 & 2.0030 & 2.4995 \\
\hline 5 & 1.23 .57 & 1.36333 & 1.5764 & 1.8712 & 2.2341 \\
\hline 6 & 1.2485 & 1.33508 & 1.5161 & 1.7329 & 1.9764 \\
\hline 7 & 1.24366 & 1.3212 & 1.4415 & 1.5868 & 1.7257 \\
\hline 8 & 1.2209 & 1.275 .3 & 1.3539 & 1.4351 & 1.4843 \\
\hline ? & 1.1830 & 1.2159 & 1.2564 & 1.2812 & 1.2549 \\
\hline 10 & 1.1330 & 1.1460 & 1.1523 & 1.1277 & 1.0391 \\
\hline 12 & 1.0056 & 0.98400 & 0.93253 & 0.82970 & 0.65183 \\
\hline 14 & $0.85 .34: 3$ & 0.80451 & 0.71006 & 0.55534 & 0.33119 \\
\hline 16 & $0.691: 34$ & $0.62: 346$ & 0.50214 & 0.32192 & 0.08994 \\
\hline 18 & 0.536665 & 0.45884 & 0.32677 & 0.14485 & -0.06528 \\
\hline 20 & 0.403354 & 0.32374 & 0.19407 & 0.02753 & -0.14399 \\
\hline$q$ & $\mu=0.6$ & $u=0.7$ & $u=0.8$ & $u=0.9$ & $u=1.0$ \\
\hline 1 & 4.8 .340 & (6.868:3 & 9.7122 & 13.6152 & 18.8542 \\
\hline 2 & 4.1905 & 5.676:3 & 7.5866 & 9.9321 & 12.6332 \\
\hline 3 & 3.6170 & +.6510 & 5.8388 & 7.0688 & 8.1249 \\
\hline 4 & 3.1011 & 3.7660 & 4.4043 & 4.8617 & 4.9166 \\
\hline 5 & 2.6316 & 2.9989 & 3.2303 & 3.1799 & 2.6894 \\
\hline 6 & 2.2011 & 2.33346 & 2.2773 & 1.9215 & 1.1991 \\
\hline 7 & 1.8078 & 1.7636 & 1.5147 & 1.0045 & 0.25483 \\
\hline 8 & 1.4518 & 1.2788 & 0.91563 & 0.35942 & -0.29441 \\
\hline 9 & 1.13330 & $0.87 \cdot 24$ & 0.45490 & -0.07303 & -0.56620 \\
\hline 10 & 0.849966 & $0 . \overline{3} 3 \bar{j} \bar{\jmath} 3$ & 0.10988 & -0.34146 & -0.64899 \\
\hline 12 & 0.38468 & $0.04: 324$ & -0.30499 & -0.53216 & -0.49268 \\
\hline 14 & 0.04888 & $-0.2+184$ & $-0.44 \pm 07$ & -0.44014 & -0.17824 \\
\hline 16 & -0.15973 & -0.35575 & -0.40144 & -0.23144 & 0.09096 \\
\hline 18 & -0.25421 & -0.34593 & -0.26929 & -0.02886 & 0.22516 \\
\hline & -0.26510 & -0.26980 & -0.12543 & 0.10202 & 0.23050 \\
\hline
\end{tabular}

TABLE 6

Values of Modified Mathieu Function Ce $(u, q)$

$\begin{array}{cccccc}q & u=0.1 & u=0.2 & u=0.3 & u=0.4 & u=0.5 \\ 1 & 1.1412 & 1.5282 & 2.2710 & 3.5374 & 5.6077 \\ 2 & 1.1563 & 1.5151 & 2.1935 & 3.3245 & 5.1202 \\ 3 & 1.1731 & 1.5042 & 2.1202 & 3.1239 & 4.6683\end{array}$


TABLE 6 (Continued)

\begin{tabular}{|c|c|c|c|c|c|}
\hline 4 & 1.1921 & 1.4957 & 2.0516 & $2.933 .3 \% 3$ & 4.2504 \\
\hline 5 & 1.2132 & 1.4897 & $1.987: 3$ & 2.7 .581 & 3.8641 \\
\hline 6 & 1.2359 & $1.48 \mathrm{j} \mathbf{j}$ & 1.9267 & 2.5910 & 3.5062 \\
\hline 7 & 1.2590 & 1.4818 & 1.8680 & 2.4318 & 3.1731 \\
\hline 8 & 1.2802 & 1.4763 & 1.8091 & 2.2778 & 2.8609 \\
\hline 9 & 1.2970 & 1.4665 & 1.7472 & 2.1263 & 2.5659 \\
\hline 10 & 1.3064 & 1.4497 & 1.6800 & 1.97 .52 & 2.2856 \\
\hline 12 & 1.2955 & 1.3884 & 1.5250 & 1.6710 & 1.7661 \\
\hline 14 & 1.2437 & 1.2906 & 1.3455 & 1.3692 & 1.3036 \\
\hline 16 & 1.1582 & 1.1651 & 1.1512 & 1.0788 & 0.90042 \\
\hline 18 & 1.0480 & 1.0205 & 0.9506 .3 & 0.80629 & 0.5567 .5 \\
\hline 20 & 0.92029 & 0.86472 & 0.75164 & 0.55842 & 0.27427 \\
\hline$q$ & $x=0.6$ & $u=0.7$ & $u=0.8$ & $u=0.9$ & $u=1.0$ \\
\hline 1 & 8.9280 & 14.1878 & 22.4276 & 35.1766 & 54.6063 \\
\hline 2 & 7.8948 & 12.0901 & 18.2886 & 27.1920 & 39.519̄ \\
\hline 3 & 6.9605 & 10.2507 & $14.79: 38$ & 20.7544 & $28.028 j$ \\
\hline 4 & 6.1173 & 8.6426 & 11.8561 & 15.6014 & 19.3790 \\
\hline 5 & 5.3573 & 7.2398 & 9.3977 & 11.5090 & 12.9574 \\
\hline 6 & 4.6719 & 6.0181 & 7.3491 & $8.287: 3$ & 8.2676 \\
\hline 7 & 4.0527 & 4.9552 & 5.6499 & 5.7767 & 4.9121 \\
\hline 8 & 3.4918 & 4.0311 & 4.2479 & 3.8446 & 2.5754 \\
\hline 9 & 2.9824 & 3.2293 & 3.0994 & $2 . .3820$ & 1.0082 \\
\hline 10 & 2.5195 & $2 . \overline{5} 361$ & 2.1678 & 1.2989 & 0.01418 \\
\hline 12 & 1.7215 & 1.4339 & $0.8: 3522$ & -0.01644 & -0.84049 \\
\hline 14 & 1.0801 & 0.65007 & 0.04279 & -0.56769 & -0.86016 \\
\hline 16 & 0.57767 & 0.11871 & -0.37198 & $-0.6 \bar{\jmath} 4: 3 \bar{\tau}$ & -0.54741 \\
\hline 18 & 0.19727 & -0.21384 & -0.52797 & $-0.54+12$ & -0.17196 \\
\hline 20 & -0.07276 & -0.38751 & -0.51169 & -0.31371 & 0.12777 \\
\hline
\end{tabular}

TABLE 7

Values of Modified Mathieu Function $\operatorname{Se}_{1}(u, q)$

$\begin{array}{rlllll}q & u=0.1 & u=0.2 & u=0.3 & u=0.4 & u=0.5 \\ 1 & .06840 & .13532 & .19914 & .25799 & .30961 \\ 2 & .04709 & .09159 & .13086 & .16208 & .18239 \\ 3 & .03295 & .06296 & .08715 & .10274 & .10727 \\ 4 & .02347 & .04404 & .05895 & .06582 & .06295 \\ 5 & .01700 & .03131 & .04047 & .04256 & .03661 \\ 6 & .01251 & .02260 & .02815 & .02771 & .02090 \\ 7 & .00934 & .01654 & .01981 & .01811 & .01152 \\ 8 & .00705 & .01224 & .01408 & .01184 & .00593 \\ 9 & .00538 & .00916 & .01009 & .00772 & .00264 \\ 10 & .00415 & .00691 & .00728 & .00499 & .00075 \\ 12 & .00253 & .00404 & .00385 & .00197 & -.0008 .3 \\ 14 & .00159 & .00242 & .00207 & .00065 & -.00111 \\ 16 & .00102 & .00149 & .00111 & .00009 & -.00098 \\ 18 & .00067 & .00093 & .00060 & -.00012 & -.00075 \\ 20 & .00044 & .00059 & .00032 & -.00018 & -.00053 \\ q & u=0.6 & u=0.7 & u=0.8 & u=0.9 & u=1.0 \\ 1 & .35130 & .37984 & .39160 & .38277 & .34981 \\ 2 & .18892 & .17923 & .15176 & .10677 & .04736 \\ 3 & .09899 & .07736 & .04382 & .00256 & -.03898 \\ 4 & .04974 & .02742 & -.00056 & -.02823 & -.04767 \\ 5 & .02305 & .00415 & -.01572 & -.03061 & -.03443\end{array}$




\begin{tabular}{rrrrrr}
\multicolumn{5}{c}{ TABLE 7 (Continued) } \\
6 & .00883 & -.00566 & -.01822 & -.02392 & -.01929 \\
7 & .00153 & -.00889 & -.01587 & -.01590 & -.00797 \\
8 & -.00197 & -.00906 & -.01215 & -.00920 & -.00104 \\
9 & -.00341 & -.00795 & -.00856 & -.00440 & .00249 \\
10 & -.00377 & -.00644 & -.00559 & -.00130 & .00381 \\
12 & -.00317 & -.00366 & -.00177 & .00138 & .00329 \\
14 & -.00219 & -.00178 & -.00004 & .00169 & .00172 \\
16 & -.00136 & -.00070 & .00054 & .00123 & .00053 \\
18 & -.00079 & -.00015 & .00061 & .00069 & -.00007 \\
20 & -.00042 & .00008 & .00049 & .00030 & -.00028
\end{tabular}

TABLE 8

Values of Modified Mathieu Function $\mathrm{Se}_{2}(u, q)$

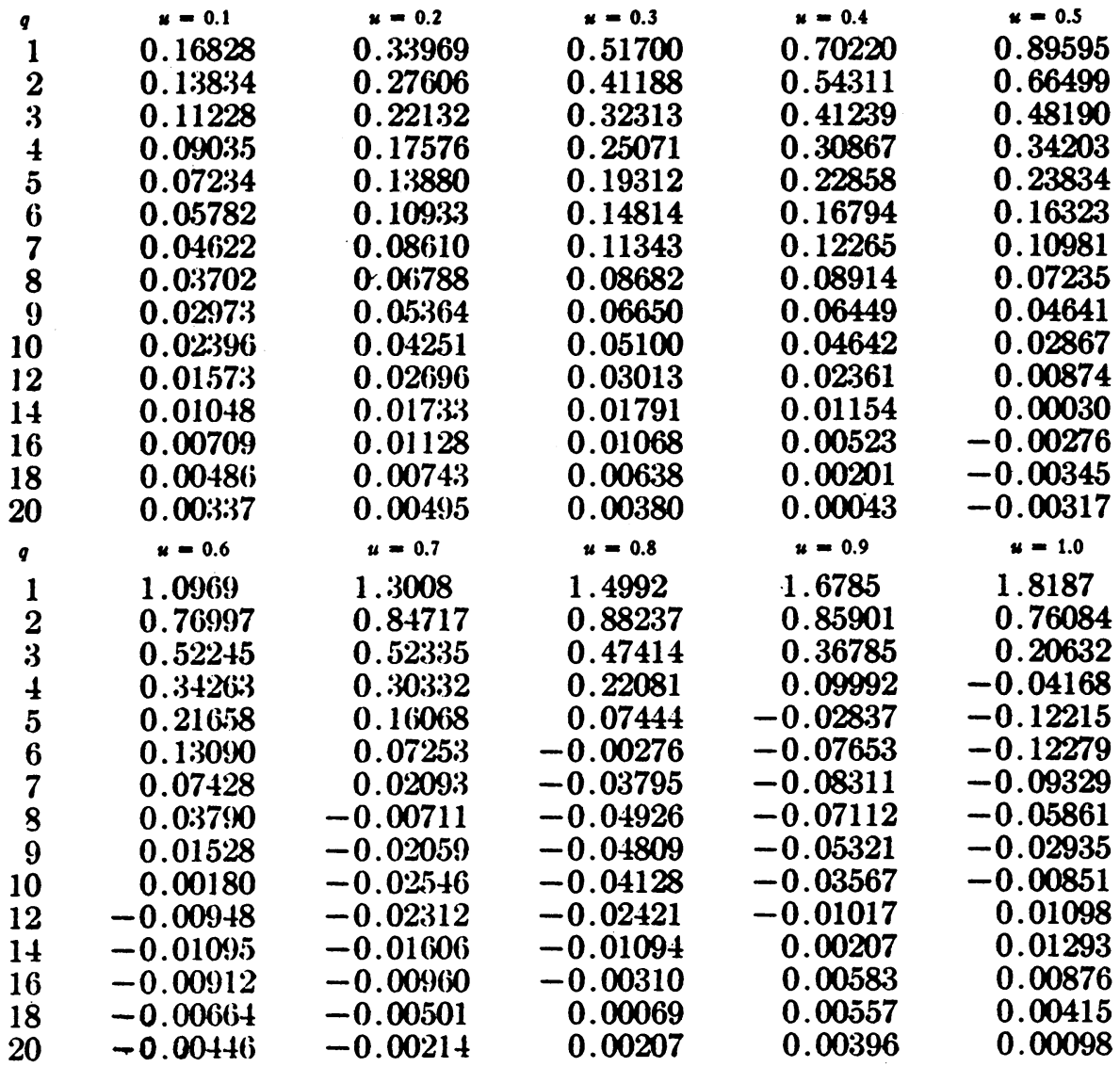

TABLE 9

Values of Modified Mathieu Function $\mathrm{Se}_{3}(u, q)$

$\begin{array}{llllll}q & u=0.1 & *=0.2 & \mu=0.3 & u=0.4 & u=0.5 \\ 1 & 0.28177 & 0.58335 & 0.92520 & 1.3289 & 1.8174 \\ 2 & 0.25585 & 0.52460 & 0.81849 & 1.1481 & 1.5208 \\ 3 & 0.22907 & 0.46514 & 0.71366 & 0.97678 & 1.2509 \\ 4 & 0.20280 & 0.40767 & 0.61469 & 0.81981 & 1.0124\end{array}$


TABLe 9 (Continued)

\begin{tabular}{|c|c|c|c|c|c|}
\hline 5 & 0.17782 & 0.35376 & 0.52376 & 0.67953 & 0.80655 \\
\hline 6 & 0.15465 & 0.30434 & 0.44202 & 0.55677 & 0.6326 \\
\hline 7 & 0.13358 & 0.25990 & 0.36990 & 0.45135 & 0.488 \\
\hline 8 & 0.11473 & 0.22060 & 0.30732 & 0.36236 & 0.371 \\
\hline 9 & 0.09810 & 0.18633 & 0.25380 & 0.28836 & 0.277 \\
\hline 10 & 0.08362 & 0.15680 & 0.20856 & 0.22765 & 0.204 \\
\hline 12 & 0.06038 & 0.11023 & 0.13930 & 0.13881 & 0.104 \\
\hline 14 & 0.04348 & 0.07713 & 0.09212 & 0.08232 & 0.04 \\
\hline 16 & 0.03134 & 0.05394 & 0.06054 & 0.04734 & 0.016 \\
\hline 18 & 0.02267 & 0.03780 & 0.03961 & 0.02616 & 0.001 \\
\hline 20 & 0.01648 & 0.02658 & 0.02582 & 0.01359 & -0.005 \\
\hline q & $x=0.6$ & $u=0.7$ & $x=0.8$ & $u=0.9$ & $u=1.0$ \\
\hline 1 & 2.4142 & 3.1416 & 4.0172 & 5.0475 & 6.2179 \\
\hline 2 & 1.9378 & 2.3902 & 2.8537 & 3.2814 & 3.5986 \\
\hline 3 & 1.5235 & 1.7698 & 1.9495 & 2.0066 & 1.8751 \\
\hline 4 & 1.1728 & 1.2712 & 1.2683 & 1.1222 & 0.8042 \\
\hline 5 & 0.88305 & 0.88107 & 0.77178 & 0.53732 & 0.1899 \\
\hline 6 & 0.64900 & 0.58420 & 0.42370 & 0.17408 & -0.1203 \\
\hline 7 & 0.46430 & 0.36502 & 0.19076 & -0.03205 & -0.2410 \\
\hline 8 & 0.32187 & 0.20848 & 0.04385 & -0.13250 & -0.2537 \\
\hline 9 & 0.21456 & 0.10082 & -0.04135 & -0.16629 & -0.2133 \\
\hline 10 & 0.13560 & 0.03006 & -0.08430 & -0.16155 & -0.1541 \\
\hline 12 & 0.03941 & -0.03848 & -0.09867 & -0.10630 & -0.0464 \\
\hline 14 & -0.00455 & -0.05363 & -0.07407 & -0.04790 & 0.0134 \\
\hline 16 & -0.02077 & -0.04703 & -0.04423 & -0.00946 & 0.0323 \\
\hline 18 & -0.02360 & -0.03432 & -0.02094 & 0.00930 & 0.0293 \\
\hline 20 & -0.02082 & -0.02216 & -0.00612 & 0.01 .502 & 0.018 \\
\hline
\end{tabular}

TABLE 10

Values of Modified Mathieu Function $\mathrm{Se}_{4}(u, q)$

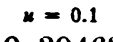

0.39463

0.37627

0.35586

0.33385

0.31081

0.28730

0.26380

0.24073

0.21842

0.19712

$0.158: 32$

0.12520

0.09787

0.07590

0.05858

$u=6.6$

4.7138

$+.0298$

3.4110

2.8560

2.3634

1.9310 $\mu=0.2$

0.84503

0.79817

0.74793

0.69530

0.64145

0.58752

0.53448

0.48314

0.43413

0.38791

0.30511

0.23597

0.18015

0.13626

0.10243

$u=0.7$

6.7818

5.5534

4.4900

3.5782

2.8050

2.1575

$u=0.3$
1.4138
1.3148
1.2133
1.1107
1.0090
0.90979
0.81449
0.72419
0.63969
0.56153
0.42530
0.31565
0.23039
0.16591
0.11819
$u=0.8$
9.6490
7.4914
5.7143
4.2677
3.1063
2.1891

$u=0.4$

2.1776

1.9819

1. 7897

1. 6029

1. $42: 39$

1. $25+7$

1.0967

0.95107

0.81833

0.69870

0. 49800

$0.3+487$

$0.232: 36$

0.15236

0.09707

$u=0.9$

13. 5682

9.8579

6.97ว9

4.7731

3.1223

1.9148 $u=0.5$

3.2347

2.8633

2.5130

2.1858

1.8836

1.6076

1.3585

1.1363

$0.9+041$

0.76968

0.49758

0.30507

0.17521

$0.0916 \mathrm{~J}$

0.04051

$u=1.0$

18.8187

12.5758

8.0609

4.8735

2.6921

1. 2588 


\begin{tabular}{rrrrrr}
\multicolumn{5}{c}{ TABLE } & 10 (Continued) \\
7 & 1.5561 & 1.6228 & 1.4782 & 1.0577 & 0.36942 \\
8 & 1.2350 & 1.1880 & 0.93945 & 0.47267 & -0.13593 \\
9 & 0.96346 & 0.84048 & 0.54207 & 0.09435 & -0.38047 \\
10 & 0.73712 & 0.56804 & 0.25870 & -0.13131 & -0.45716 \\
12 & 0.40088 & 0.20320 & -0.05883 & -0.29067 & -0.35966 \\
14 & 0.18811 & 0.01168 & -0.16636 & -0.25361 & -0.17308 \\
16 & 0.06334 & -0.07176 & -0.16852 & -0.15892 & -0.02863 \\
18 & -0.00295 & -0.09442 & -0.12925 & -0.07123 & 0.04656 \\
20 & -0.03308 & -0.08730 & -0.08258 & -0.01164 & 0.06763
\end{tabular}

TABLE 11

Values of Modified Mathieu Function $\operatorname{Se}_{5}(u, q)$

$x=0.1$

0.50806

0.49404

0.47893

0.46266

0.44522

0.42665

0.40710

0.38675

0.36580

0.34451

0.30177

0.26020

0.22112

0.18547

0.15383

$u=0.6$

\subsection{0}

7.8187

6.8646

5.9972

5.2102

4.4984

3.8571

3.2821

2.7695

2.3154

1.5673

1.0064

0.60145

0.32205

0.13950 $u=0.2$
1.1350

1.1350

1.0935

1.0055

0.95900

0.91094

0.86162

0.81145

0.76086

0.71035

0.61133

0.51760

0.43160

0.35491

0.28828

$u=0.7$

14.1488

12.0384

10.1820

8.55:32

7.1291

5.8894

4.8161

3.8927

3.1041

2. 4362

1.4110

0.72162

0.28547

0.03208

$-0.09651$
$*=0.3$
2.0261

2.0261

1.9233

1.8205

1.7175

1.6143

1.5113

1. 4089

1.3076

1. 2082

1.1113

0.92761

0.76060

0.61301

0.48605

0.37963

$u=0.8$

22.4024

18.2527

14.7432

11.7889

9.3159

7.2595

5.5633

4.1773

3.0573

2.1638

0.92094

0.21470

$-0.13573$

$-0.26611$

$-0.27382$ $u=0.4$

3.3842

3.1498

2.9229

2.7032

2.4904

2.2847

2.0865

1.8965

1.7154

1.5437

1. 2307

0.96037

0.73302

0.54707

0.39910

$u=0.9$

35.1601

27.1664

20.7166

15.5516

11.4525

8.2343

5.7400

3.8368

2.4121

1. 3709

0.13225

$-0.37207$

$-0.47604$

$-0.39470$

$-0.25559$ $u=0.5$

5.5114

5.0058

4.5311

4.0855

3.6675

3.2764

2.9116

2.5728

2. 2599

1.9724

1.4718

1.0654

0.74519

0.50073

0.32040

54.5952

39.5007

28.0002

19.3442

12.9247

8.2493

4.9202

2.6181

1.0872

0.12471

$-0.69719$

$-0.73439$

$-0.48918$

$-0.21558$

$-0.01570$

TABLE 12

Values of Modified Mathieu Function $\mathrm{Se}_{6}(u, q)$

$$
u=0.1
$$

0.62524

0.61333

0.60091

0.58792

0.57431

$u=0.2$

1.4688

1.4278

1.3863

1.3442

1.3015

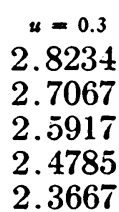

$u=0.3$
2.8234
2.7067
2.5917
2.4785
2.3667

$4=0.4$

5.1539

4.8536

4.5649

4.2872

4.0198 $u=0.5$
9.2485
8.5252
7.8454
7.2067
6.6067 
Table 12 (Continued)

$\begin{array}{rccccc}6 & 0.56004 & 1.2581 & 2.2562 & 3.7624 & 6.0431 \\ 7 & 0.54506 & 1.2138 & 2.1469 & 3.5143 & 5.5139 \\ 8 & 0.52935 & 1.1687 & 2.0387 & 3.2753 & 5.0174 \\ 9 & 0.51290 & 1.1227 & 1.9317 & 3.0452 & 4.5517 \\ 10 & 0.49573 & 1.0760 & 1.8258 & 2.8237 & 4.1161 \\ 12 & 0.45945 & 0.98057 & 1.6184 & 2.4068 & 3.3294 \\ 14 & 0.42114 & 0.88388 & 1.4184 & 2.0256 & 2.6494 \\ 16 & 0.38169 & 0.78772 & 1.2283 & 1.6812 & 2.0693 \\ 18 & 0.34208 & 0.69405 & 1.0507 & 1.3748 & 1.5822 \\ 20 & 0.30326 & 0.60465 & 0.88754 & 1.1066 & 1.1806 \\ q & \mathbf{q}=0.6 & u=0.7 & u=0.8 & \kappa=0.9 & u=1.0 \\ 1 & 16.4676 & 29.1545 & 51.3078 & 89.6491 & 155.25 .5 \\ 2 & 14.7990 & 25.4199 & 43.1373 & 72.1129 & 118.287 \\ 3 & 13.2690 & 22.0897 & 36.0874 & 57.5680 & 89.0684 \\ 4 & 11.8672 & 19.1253 & 30.0221 & 45.5612 & 66.1482 \\ 5 & 10.5841 & 16.4914 & 24.8206 & 35.7016 & 48.3227 \\ 6 & 9.4108 & 14.1560 & 20.3757 & 27.6527 & 34.5956 \\ 7 & 8.3393 & 12.0899 & 16.5923 & 21.1260 & 24.1462 \\ 8 & 7.3621 & 10.2671 & 13.3865 & 15.8741 & 16.3000 \\ 9 & 6.4725 & 8.6636 & 10.6839 & 11.6855 & 10.5056 \\ 10 & 5.6645 & 7.2582 & 8.4189 & 8.3797 & 6.3134 \\ 12 & 4.2713 & 4.9650 & 4.9760 & 3.8237 & 1.3490 \\ 14 & 3.1428 & 3.2514 & 2.6685 & 1.2321 & -0.73178 \\ 16 & 2.2438 & 2.0028 & 1.1913 & -0.09221 & -1.28226 \\ 18 & 1.5421 & 1.1221 & 0.30627 & -0.63917 & -1.12283 \\ 20 & 1.0078 & 0.52675 & -0.17078 & -0.74531 & -0.72343\end{array}$

